\title{
Descripción de casos registrados en el Centro Nacional de Toxicología (MSPyBS) por exposición debida a incendio de transformadores fuera de uso
}

\author{
*Stella Presentado de Núñez ${ }^{1}$, Lis Kreitmayr ${ }^{1}$, José Antonio Núñez P. ${ }^{2}$ \\ ${ }^{1}$ Centro Nacional de Toxicología, Ministerio de Salud Pública y Bienestar Social, Paraguay \\ ${ }^{2}$ Grupo de Investigación en Ingeniería Biomédica, Departamento de Investigación y PostGrado, Facultad \\ Politécnica, Universidad Nacional de Asunción, Paraguay
}

Cómo referenciar este artículo/

How to reference this article:
Presentado de Núñez S, Kreitmayr L, Núñez J. Descripción de casos registrados en el Centro Nacional de Toxicología (MSPyBS) por exposición debida a incendio de transformadores fuera de uso. Mem. Inst. Investig. Cienc. Salud. 2018; 16(1): 71-77

\section{R E S U M E N}

En octubre de 2015 el depósito de transformadores fuera de uso de la Administración Nacional de Electricidad (ANDE) ubicado en San Lorenzo (Paraguay), sufrió un incendio de gran magnitud, con destrucción de transformadores, condensadores, y otros materiales que pudieron contener bifenilopoliclorados (PCBs), considerados compuestos orgánicos persistentes, de baja toxicidad aguda, clasificados como cancerígenos (Grupo1) por la Agencia Internacional para la Investigación sobre el cáncer (IARC). Niveles de PCBs en plasma son considerados biomarcadores de exposición al agente químico. El objetivo del trabajo fue describir los casos toxicológicos por exposición al incendio de transformadores fuera de uso, según síntomas manifestados, actividades relacionadas al incendio y resultados del biomarcador de exposición. En este estudio observacional descriptivo de corte transversal, se estudiaron 190 historias clínicas del Centro Nacional de Toxicología (CNTox), hasta 29 días posteriores al incendio, que contaban con resultados del biomarcador determinado por GC/MS. Se detectó el biomarcador hasta 13 días después del incendio. Se observaron niveles plasmáticos de PCBs: $<1 \mathrm{ng} / \mathrm{ml}$ en $86,84 \%(165 / 190)$ y $\geq 1$ $\mathrm{ng} / \mathrm{ml} 13,16 \%(25 / 190)$. No se registraron casos de cloracné. Pacientes asintomáticos constituyeron 48,94\% (93/190), con detección del biomarcador en 56\% (14/25). Pacientes con síntomas constituyeron 51,06\% (97/190) con detección del biomarcador en $44 \%$ $(11 / 25)$. Teniendo en cuenta la actividad de los pacientes con PCB detectado en plasma fueron bomberos $88 \%(22 / 25)$. Pacientes asintomáticos con niveles detectables de PCBs en plasma como biomarcador de exposición, describe la complementación de datos clínicos con los laboratoriales.

Palabras clave: síntomas, biomarcador de exposición, PCBs.

\section{Description of cases registered at the National Toxicology Center (MSPyBS) for exposure due to a fire of discarded transformers}

\section{A B S T R A C T}

In October 2015, the warehouse of discarded transformers of the National Electricity Administration (ANDE) located in San Lorenzo (Paraguay) caught fire destroying transformers, capacitors, and other materials that could contain polychlorinated biphenyls (PCBs), which are considered persistent organic compounds of low acute toxicity, classified as carcinogenic (Group 1) by the International Agency for Research on Cancer (IARC). Levels of PCBs in plasma are biomarkers of exposure to these chemical agents. The objective of the study was to describe toxicological cases due to exposure to the burned 
discarded transformers, according to the declared symptoms, fire-related activities and exposure biomarker results. In this descriptive cross-sectional observational study, 190 clinical records of the National Toxicology Center (CNTox) were studied, until 29 days after the fire, with biomarker results determined by GC/MS. The biomarker was detected until 13 days after the fire. The following plasmatic levels of PCBs were observed: $<1 \mathrm{ng} / \mathrm{ml}$ in $86.84 \%(165 / 190)$ and $\geq 1 \mathrm{ng} / \mathrm{ml} 13.16 \%$ (25/190). There was no record of chloracne cases. Asymptomatic patients were $48.94 \%(93 / 190)$, with biomarker detection in $56 \%$ $(14 / 25)$. Patients with symptoms were $51.06 \%$ (97/190) with detection of the biomarker in $44 \%(11 / 25)$. Taking into account the activity of patients with PCB detected in plasma, it was found that they were mostly firefighters $88 \%(22 / 25)$. The asymptomatic patients with detectable levels of PCBs in plasma as an exposure biomarker describe the complementation between clinical and laboratory data.

Keywords: symptoms, exposure biomarker, PCBs.

\section{INTRODUCCIÓN}

El 14 de octubre de 2015 pasadas las 17:00 horas de Paraguay el depósito de transformadores averiados y fuera de uso de la Administración Nacional de Electricidad ubicado San Lorenzo (Paraguay), sufrió un incendio de gran magnitud. Se estima que transformadores, condensadores y otros equipos almacenados en ese lugar podían contener bifenilopoliclorados (PCBs), los cuales podrían haber generado una contaminación por PCBs, sus derivados, dioxinas y furanos ${ }^{(1)}$. Desde ese mismo día acudieron al Centro Nacional de Toxicología (CNTox) los pacientes expuestos.

Los PCBs son mezclas de diversos bifenilos clorados, contabilizándose hasta 209 compuestos análogos siendo la principal aplicación como refrigerante en trasformadores de energía y capacitadores. Su fórmula molecular es $\mathrm{C}_{12} \mathrm{H}_{10-n} \mathrm{Cl}_{n}$, donde $\mathrm{n}$ es mayor que 1 . Fueron producidos por cerca de 50 años entre 1929 y $1977^{(2-6)}$. Los PCBs son insolubles en agua, lipofílicos, refractarios a la biodegradación y persistentes en el ambiente con propiedades de bioacumulación y biomagnificación ${ }^{(4-6)}$. Son considerados contaminantes orgánicos persistentes por lo que su eliminación está recomendada por el Convenio de Estocolmo ${ }^{(7)}$. Las vías de exposición, desde el punto de vista ocupacional son con frecuencia la inhalación y la dérmica. Para la población en general puede ser dérmica, inhalatoria y oral por el consumo de alimentos considerados potenciales fuentes de PCBs como: huevos, derivados lácteos, grasas de animal, algunos peces y animales de vida silvestre ${ }^{(4,8)}$.

Una vez absorbido los PCBs, se distribuyen, luego van al hígado y tejido muscular, son metabolizados en el hígado para luego ser excretados por heces y orina. En general, los congéneres de PCBs menos clorados se metabolizan más fácilmente que los congéneres altamente clorados. Por lo tanto, los congéneres altamente clorados tienden a concentrarse más en los tejidos adiposos, donde se almacenan en forma solubilizada ${ }^{(4)}$.

Los PCBs tienen baja toxicidad aguda, pero conciernen a la salud pública porque pueden producir afecciones por exposición crónica o a largo plazo ${ }^{(4,6,8,9)}$.

Por exposición a PCBs se han registrado síntomas por exposición aguda tales como: irritación de ojos, cloracné, afectando principalmente los siguientes órganos: ojos, hígado. Además de efectos adversos sobre sistema nervioso periférico, sistema digestivo, sistema reproductivo, sistema inmune ${ }^{(4,10,11)}$.

Los PCBs son potencialmente capaces de producir disrupción endócrina y hepatopatías. Fueron clasificados por la IARC en el Grupo 1, carcinógenos para humanos. La clasificación está basada en la relación entre exposición a PCBs y riesgos crecientes de melanoma en humanos $^{(12)}$.

Entre sus metabolitos están los que se unen a receptores de estrógeno y tienen capacidad para inducir una respuesta estrogénica, y otros como OH-PCBs tienen capacidad antiestrogénica e inhiben la respuesta inducida por los estrógenos, estos últimos compiten con las hormonas tiroideas en su unión a su principal proteína sérica de transporte, la transtiretina ${ }^{(13-15)}$.

Los contaminantes se evalúan más a fondo en la evaluación de efectos de salud. Los resultados de la evaluación de exposición pueden también conducir a pruebas biomédicas adicionales para determinar dosis de exposición y niveles de carga de cuerpo en la población expuesta u otros estudios de epidemiológicos ${ }^{(16)}$. 
El término biomarcador permite reflejar las interacciones entre el sistema biológico y el agente ambiental (químico, físico o biológico). Se considera biomarcador de exposición a la sustancia exógena o su metabolito o el producto de una interacción entre el agente xenobiótico con alguna molécula o célula diana que puede ser medido en una muestra biológica. Un marcador biológico además ofrece una evidencia directa de la exposición de los individuos a una sustancia específica. Es necesario conocer la información respecto a la cinética del agente químico pues va disminuyendo la detección en relación al tiempo posterior de exposición. La detección de niveles de PCBs en plasma es considerada un biomarcador de exposición ${ }^{(17)}$.

Los métodos analíticos utilizados generalmente no difieren para el suero y el plasma. Niveles de PCBs sérico $>20 \mathrm{ppb}$, se consideran tóxicos aunque otros autores consideran mayores a 200 ppb en exposiciones laborales ${ }^{(4,18)}$.

Los niveles de PCBs en suero pueden no tener coherencia con los efectos adversos en la salud. Es difícil relacionar la presencia de cualquier signo-síntoma o anormalidad laboratorial, con la posible excepción del cloracné, con la exposición absoluta a PCBs en cualquier paciente dado. Sin embargo, se han reportado asociaciones estadísticamente significativas entre los efectos dermatológicos y los niveles plasmáticos de congéneres de PCB clorados superiores ${ }^{(5)}$.

Las ocupaciones específicas con riesgo de exposición a PCBs en la Encuesta Nacional de Exposición Ocupacional (NOES) [NIOSH 1989] incluyen: trabajos de construcción, trabajos de demolición, reparación de cables eléctricos, galvanoplastia, respuesta ante emergencias, lucha contra incendios, transporte de residuos peligrosos o funcionamiento del sitio, reparación de equipos de intercambio térmico, mantenimiento o limpieza, laboratorio médico, fabricación de productos de madera, reparación de transformadores o condensadores, procesamiento de aceites usados ${ }^{(4)}$.

Las metodologías analíticas recomendadas para determinación de PCBs en plasma son cromatografía de gases de alta resolución, utilizando como detector captura de electrones HRGC/ECD o técnicas de espectrometría de masas (HRGC / MS) ${ }^{(12,19)}$.

El objetivo del trabajo fue describir los casos toxicológicos por exposición al incendio de transformadores fuera de uso, según síntomas manifestados, actividades relacionadas al incendio y resultados del biomarcador de exposición.

\section{MATERIALES Y MÉTODOS}

En este estudio observacional descriptivo de corte transversal, se observaron 190 historias clínicas de pacientes, de 8 a 65 años de edad, que consultaron en el Centro Nacional de Toxicología (Ministerio de Salud Pública y Bienestar Social) y cuyas muestras de sangre fueron recolectadas entre el 15 de octubre y el 12 de noviembre de 2015 para determinar niveles de PCBs en plasma, considerados biomarcadores de exposición.

Se guardó la confidencialidad de los datos personales, los datos clínicos encontrados y resultados laboratoriales.

La determinación de PCBs en plasma no tuvo costo para los pacientes, fue abonada por diversas entidades gubernamentales (Secretaria de Emergencia Nacional, ANDE, MSPyBS). Se realizó en laboratorio privado (LE003) acreditado por el Organismo Nacional de Acreditación según Norma -ISO/IEC 17025:2006, al igual que el procedimientos de recolección de muestras (TOX-PRT-005 V4) y la determinación de Bifenilos policlorados en sangre y orina por GC / MS (TOX-PRT-002 V3). El valor de referencia para población normal no expuesta considerado fue: hasta $20 \mathrm{ng} / \mathrm{ml}$ y el límite de cuantificación: $1 \mathrm{ng} / \mathrm{ml}$. En este trabajo se consideran los resultados expresados como PCBs totales, siendo los congéneres de PCBs investigados: 28, 52, 49, 44, 74, 70, 66, 60, 77, 101, 99, 87, 82, 105, 114, 118, $126,153,138,158,166,156,128,169,179,187,180,183,170,189^{(20)}$.

\section{Las variables fueron referidas a:}

-Síntomas por exposición aguda a PCBs: Asintomáticos, Síntomas dérmicos y/o mucosas; Síntomas dérmicos y/o mucosas acompañado por otros síntomas (cefalea, mareos, síntomas digestivos); Síntomas digestivos con o sin cefalea; Cefalea y/o mareos; Neuropatías de tipo periférico.

-Niveles de PCBs en plasma: Inferiores a $1 \mathrm{ng} / \mathrm{ml}$; entre 1 y 9,4 ng/ml; 28,3ng/ml; -Tiempo de detección del biomarcador de exposición después del evento: días; 
-Actividad relacionada en el momento del evento: bomberos, funcionarios de ANDE, residentes, policías, guardias de seguridad, otros (incluye a pacientes que se encontraban casualmente en las inmediaciones).

Para el análisis y procesamiento de datos se utilizaron los programas Microsoft Excel y Word.

\section{RESULTADOS}

De la población de 190 pacientes, se tomaron las muestras de sangre total para la detección de niveles plasmáticos de PCBs, obteniéndose niveles detectables hasta 13 días después del incendio.

Tabla 1. Toma de muestra y detección de PCBs en plasma en relación al tiempo transcurrido a partir del incendio

\begin{tabular}{ccc}
\hline $\begin{array}{c}\text { Toma de muestra posterior } \\
\text { al incendio (días) }\end{array}$ & $\begin{array}{c}\text { Pacientes } \\
\text { expuestos }(\mathbf{n = 1 9 0 )}\end{array}$ & $\begin{array}{c}\text { Pacientes con niveles } \\
\text { detectables de PCBs (n=25) }\end{array}$ \\
\hline 1 & 3 & 0 \\
2 & 1 & 0 \\
3 & 1 & 0 \\
4 & 1 & 1 \\
5 & 2 & 0 \\
8 & 65 & 17 \\
9 & 13 & 1 \\
10 & 2 & 0 \\
12 & 4 & 3 \\
13 & 12 & 3 \\
14 a 29 & 86 & 0 \\
\hline
\end{tabular}

Los resultados de niveles plasmáticos de PCBs entre 1 y $9,4 \mathrm{ng} / \mathrm{ml}$ se dieron en $12,63 \%$ (24/190), mientras que resultados inferiores a $1 \mathrm{ng} / \mathrm{ml}$ se dieron en $86,84 \%(165 / 190)$, valores mayores a $20 \mathrm{ng} / \mathrm{ml}$ se observó en 0,53\% (1/190). La distribución de los rangos en $\mathrm{ng} / \mathrm{ml}$ se muestra en la Figura 1.

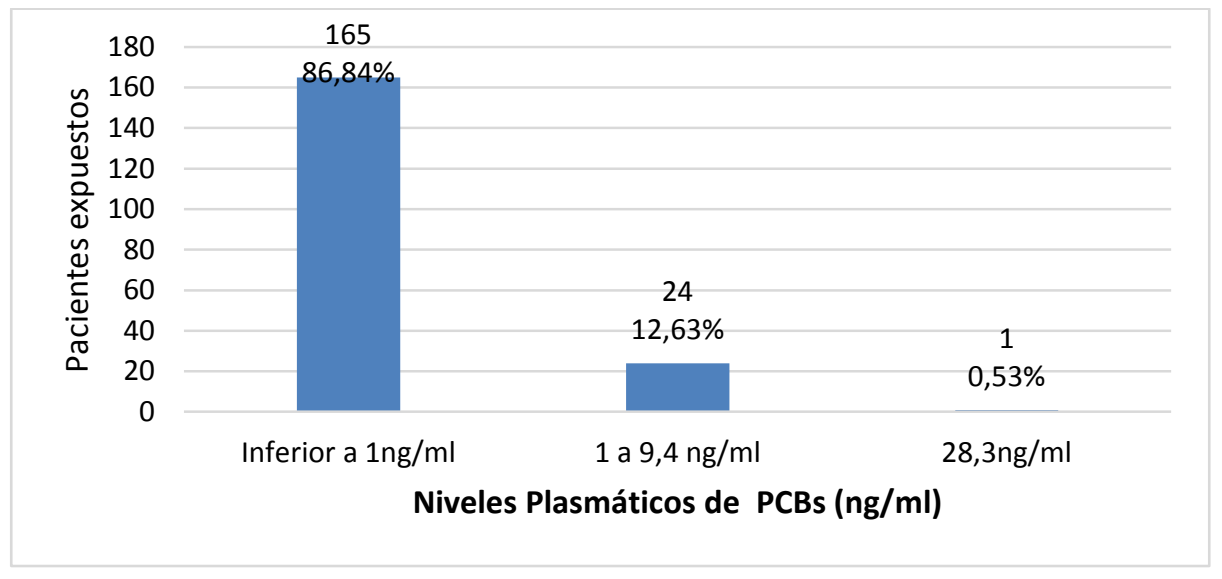

Figura 1. Distribución de rangos de niveles plasmáticos de PCBs en plasma

En relación a los síntomas, no se observaron casos de cloracné. Se presentaron: asintomáticos en $48,94 \%(93 / 190)$ y con síntomas 51,06\% (97/190), de tipo: dérmico (prurito, eritema o rash cutáneo), síntomas en mucosas (irritación de: ojos y/o garganta y/o fosas nasales), síntomas respiratorios (dificultad respiratoria, bronco espasmo), síntomas digestivos (náuseas, vómitos, diarrea, dolor abdominal), cefalea y/o mareos, neuropatías de tipo periférico (temblores, parestesias). La relación entre signos y síntomas con los niveles plasmáticos de PCBs se observa en la Tabla 2. 
Tabla 2. Síntomas y signos relacionados con detección de niveles plasmáticos de PCBs

\begin{tabular}{|c|c|c|c|c|}
\hline Síntomas-signos & $\begin{array}{l}\text { Población } \\
\text { estudiada } \\
(n=190)\end{array}$ & $\%$ & $\begin{array}{l}\text { PCBs detectados } \\
\text { en plasma } \\
(n=25)\end{array}$ & $\%$ \\
\hline Asintomáticos & 93 & 48,94 & 14 & 15 \\
\hline $\begin{array}{l}\text { Síntomas dérmicos y/o en } \\
\text { mucosas }\end{array}$ & 34 & 17,9 & 4 & 11,2 \\
\hline $\begin{array}{lr}\text { Síntomas dérmicos y/o mucosas } \\
\text { acompañado por } & \text { otros } \\
\text { síntomas(cefalea, } & \text { mareos, } \\
\text { síntomas digestivos) } & \end{array}$ & 25 & 13,16 & 2 & 8 \\
\hline $\begin{array}{l}\text { Síntomas digestivos con o } \sin \\
\text { cefalea }\end{array}$ & 13 & 6,84 & 2 & 15,4 \\
\hline Cefalea y/o mareos & 12 & 6,32 & 2 & 8 \\
\hline $\begin{array}{l}\text { Síntomas respiratorios con o sin } \\
\text { síntomas en mucosas }\end{array}$ & 11 & 5,79 & 1 & 4 \\
\hline Neuropatías de tipo periférico & $\begin{array}{c}2 \\
190\end{array}$ & $\begin{array}{l}1,05 \\
100\end{array}$ & $\begin{array}{c}0 \\
25\end{array}$ & $\begin{array}{l}0 \\
100\end{array}$ \\
\hline
\end{tabular}

En la Tabla 3 se clasifica la población estudiada y la población de pacientes con PCBs detectado en plasma según su actividad en el momento del incendio.

Tabla 3. Actividad en relación al incendio con detección de PCBs en plasma

\begin{tabular}{lllll}
\hline Actividad & $\begin{array}{l}\text { Población } \\
\text { estudiada } \\
\text { (n=190) }\end{array}$ & \% & $\begin{array}{l}\text { Pacientes con PCBs } \\
\text { detectado en } \\
\text { plasma(n=25) }\end{array}$ & \% \\
\hline Bomberos & 99 & 52,11 & 22 & 88 \\
Func. de ANDE & 60 & 31,58 & 0 & 0 \\
Guardias de seguridad & 8 & 4,20 & 0 & 0 \\
Policías & 9 & 4,74 & 0 & 0 \\
Residentes & 11 & 5,79 & 3 & 12 \\
Otros & 3 & 1,58 & 0 & 0 \\
Total & 190 & 100 & 25 & 100 \\
\hline
\end{tabular}

\section{DISCUSIÓN}

Los niveles en PCBs en plasma fueron detectados hasta 13 días después del incendio, los que implica las limitaciones en la detección del biomarcador con el transcurso del tiempo ya sea porque son eliminados o porque se han almacenados en otros compartimientos biológicos, como los ricos en lípidos. Martin-Jiménez y Hansen, 2008 en un estudio sobre PCBs como los encontrados en el aire de los edificios, en las ciudades en un día caluroso y cerca de los sitios de desecho, concluyeron que son generalmente mucho más lábiles, son susceptibles al ataque metabólico y pueden desaparecer de la sangre con vidas medias de menos de 30 días, estos congéneres de corta duración han sido denominados "episódicos"(12).

Hay pocos estudios registrados relativos a la exposición de PCBs después de accidentes. Elo et al. (1985) investigaron las consecuencias para la salud de la exposición a altos niveles de PCBs debido a la explosión de varios condensadores. Tres días después de la explosión, el nivel de PCBs en suero de los trabajadores que hacían la limpieza y descontaminación del lugar era de $30 \mathrm{ng} / \mathrm{ml}$. Probablemente estos trabajadores también habían estado expuestos a los dibenzofuranos clorados durante la limpieza, a lo cual pudo haberse atribuido los efectos en la salud. Los síntomas agudos en estos trabajadores fueron irritación de la nariz, ojos, piel, y tracto respiratorio, y síntomas generales como náuseas, dolor de cabeza y vértigo. Después de la explosión, algunos trabajadores experimentaron parestesia en las extremidades ${ }^{(1)}$. En otro evento Stark et al. (1986) publicaron un estudio de 52 sujetos expuestos a un derrame de PCBs en un edificio de oficinas que resultó de la explosión de un transformador, sin fuego posterior. Los exámenes de laboratorio, incluyendo el de enzimas hepáticas, resultaron comunes y corrientes: el nivel medio de PCBs en suero fue de $7,0 \mathrm{ng} / \mathrm{ml}$ en los sujetos expuestos. La formación de dioxinas en el momento de la explosión es factible. Se asociaron a la exposición a los PCBs irritación de la 
piel temporal y enrojecimiento ${ }^{(1)}$. Kreiss $(1985)$ encontró concentraciones séricas medias en la población no expuesta ocupacionalmente en EE.UU., entre 4 y $8 \mathrm{ng} / \mathrm{ml}$, con $95 \%$ de los individuos con concentraciones séricas de PCB inferiores a $20 \mathrm{ng} / \mathrm{ml}{ }^{(2)}$. En este trabajo la población estudiada estuvo expuesta en una explosión al aire libre, niveles detectables de PCBs en plasma en mayoría correspondieron a pacientes asintomáticos, lo que demuestra la importancia de la complementación entre datos clínicos y laboratoriales. No se observaron casos de cloracné, que es una característica de intoxicación aguda por PCBs y otros organoclorados, pero la detección en plasma del biomarcador indica la exposición a PCBs.

Dentro de la actividades durante el incendio, los resultados de niveles plasmáticos de PCBs obtenidos muestran que los bomberos fueron los pacientes más expuestos, quienes trabajaron cerca de 6 horas en la zona del siniestro, Kelly et al., 2002 consideran a los bomberos como una de las ocupaciones relacionadas a la exposición a PCBs pues son los primeros respondedores a incidentes donde transformadores han explotado ${ }^{(4)}$. Otra forma de exposición laboral es por materiales que contienen PCBs, en sitios de desechos peligrosos, limpieza de sitios de desechos tal como han manifestaron Luotamo et al., 1993; Robertson y Ludewig $2011^{(4)}$.

Se recomienda el seguimiento de los pacientes expuestos, con estudios clínicos y laboratoriales para detectar PCBs en otras muestras biológicas, como tejido adiposo, en vista a reducir consecuencias negativas en la salud.

\section{AGRADECIMIENTOS}

A los médicos del Centro Nacional de Toxicología (CNTox) por la capacidad de respuesta profesional, ante emergencias químicas como la estudiada en este trabajo.

\section{REFERENCIAS BIBLIOGRÁFICAS}

1. Environmental Emergencies Centre [sede web] Ginebra: Unidad Conjunta para el Medio Ambiente PNUMA/OCHA (JEU); 2015. Apoyo técnico por el incendio de la estación eléctrica de almacenamiento de equipos con bifenilos policlorados (PCB) San Lorenzo (Paraguay) Noviembre de 2015. Disponible en: http://eecentre.org/Modules/EECResources/Uplo adFile/Attachment/Reporte_Paraguay_Final.pdf 2. Chemical Safety Information from intergovernmental Organization[Sede Web]. Canada: International Program on Chemical Safety;2010 [acceso 14 de marzo de 2017]. Environmental health criteria 140. Polychlorinated biphenyls and terphenyls,2a edition. Disponible en: http://www.inchem.org/documents/ehc/ehc/ehc1 40.htm\#2.3.4

3. Spiro TG, Stigliani WM. Química medioambiental. $2^{\text {da }}$ ed. Madrid: Pearson Prentice Hall.; 2004: 435-6.

4. Agency for Toxic Substances and Disease Registry [Sede Web]. Atlanta:_U.S. Department of Health and Human Services; 2010 [acceso 14 de marzo de 2017].Case Study in Enviromental Medicine Polychlorinate Biphenyls (PCBs) Toxicity Disponible en: https://www.atsdr.cdc.gov/csem/pcb/docs/pcb.p df

5. U.S. Environmental Protection Agency [Sede Web]. Washington: United State Environmental Protection Agency; 2010 [acceso 14 de marzo de 2017]. Understanding PCB Risks at the GEPittsfield/Housatonic River Site Disponible en: https://www.epa.gov/ge-

housatonic/understanding-pcb-risks-gepittsfieldhousatonic-river-site\#WhatArePCBs
6. Moreno MD. Toxicología ambiental. Evaluación de riesgo para la salud humana. Madrid: Mc Graw Hill; 2003.

7. Stockholm Convention [Sede Web]. Châtelaine: Stockholm Convention. [acceso 14 de marzo de 2017]. Convenio de estocolmo sobre contaminantes orgánicos persistentes. 2008. Disponible en: http://www.pops.int/documents/convtext/convte xt_sp.pdf

8. Agency for Toxic Substances and Disease Registry [Sede Web]. Atlanta: U.S. Department of Health and Human Services;2010 [acceso 14 de marzo de 2017]. Resúmenes de Salud Pública - Bifenilos policlorados (BPCs) [Polychlorinated Biphenyls (PCBs)]. Disponible en: https://www.atsdr.cdc.gov/es/phs/es_phs17.htm I

9. Centers for Disease Control and Prevention [Sede Web]. Atlanta: National Institute for Occupational Safety and Health; 2007 [acceso 14 de marzo de 2017]. Niosh pocket guide to chemical hazards. Disponible en: https://www.cdc.gov/niosh/docs/2005-

149/pdfs/2005-149.pdf

10. Occupational Safety and Health Adminitration [Sede Web]. Washington: Occupational Safety and Health Adminitration; 2007 [acceso 14 de marzo de 2017]. Chlorodiphenyl (54\% Cl). Disponible

en: https://www.osha.gov/dts/chemicalsampling/dat a/CH_227500.html

11. Ladrón de Guevara V, Moya Pueyo V. Toxicología Médica, Clínica y Laboral. $1^{\text {a }}$ ed en español. Madrid: Interamericana, Mc Graw- HillInteramericana; 1995.

12. IARC Working Group on the Evaluation of Carcinogenic Risks to Humans. IARC monographs 
on the evaluation of carcinogenic risks to humans polychlorinated biphenyls and polybrominated biphenyls volume 107 OMS,Lyon France (monografía en Internet); 2015 [acceso 14 de marzo de 2017]. Disponible en: http://monographs.iarc.fr/ENG/Monographs/vol1 07/mono107.pdf

13. Salguero M. "Disruptores Endocrinos". Postgrado en Toxicología -09. M. Repetto (ed.). Ilustre Colegio Oficial de Químicos. Sevilla; CDROM 2009.

14. Klaassen C, Waltkins JB, Casarett y Doull. Fundamentos de Toxicología. Madrid: Mc GrawHill Interamericana; 2005.

15. Reppeto Jiménez M, Reppeto Kuhn G. Toxicologia Fundamental. 4ta Edición. Madrid: Diaz de Santos; 2009.

16. García-Repetto R, Repetto M. Evaluación Toxicológica y de riesgos específicos; Postgrado de Toxicología. M. Repetto (ed). Ilustre Colegio Oficial de Químicos. Sevilla. CD-ROM. 2008.

17. International Program on Chemical Safety. Environmental health criteria 155. Biomarkers and Risk Assessment: Concepts and Principles. Geneva: World Health Organization; 1993.

18. Repetto MR, Repetto M. Tabla de concentraciones de xenobióticos en fluidos biológicos humanos como referencia para el diagnóstico toxicológico (actualización 2007). En: "Ampliación de Toxicología de Postgrado", M Repetto, ed. Area de Toxicología. Universidad de Sevilla. CD-Rom. Sevilla; 2007.

19. World Health Organization. WHO Library Cataloguing-in-Publication Data Polychlorinated biphenyls: Human health aspects. Geneva, 2003.

(Concise international chemical assessment document; 55) [acceso 14 de marzo de 2017]. Disponible en: http://www.inchem.org/documents/cicads/cicads /cicad55.htm\#8.1

20. Consejo Nacional de Ciencias y Tecnología [sede web]. Asunción: Consejo Nacional de Ciencias y Tecnología [acceso 14 de marzo de 2017].Organismo Acreditado por el ONA. Diaz Gill Medicina Laboratorial S.A. Disponible en: http://www.conacyt.gov.py/sites/default/files/Fic ha-Diaz-Gill-Ampliacion-de alcance.pdf 